\title{
生物多样性数据集成模式初探
}

\author{
乔慧捷 ${ }^{12}$ 韩 艳 $^{1}$ 李 诺 $^{1}$ 纪力强 ${ }^{1}$ * \\ 1 (中国科学院动物研究所, 北京 100080 ) \\ 2 (中国科学院研究生院, 北京 100039)
}

摘要: 本文以生物多样性研究发展现状的分析为基础, 为生物多样性保护政策的制定提供可靠的数据支持为目标, 通过对国内外几个著名的生物多样性数据库建设情况的分析, 从相关学者的需求出发, 提出了设计一个多层次多 角度并带有一定人工智能的生物多样性集成数据库的构想。该系统基于都柏林核心 (Dublin Core) 的数据规范, 并 符合基于开放文献预研的元数据互操作协议 (The Open Archives Initiative Protocol for Metadata Harvesting, OAI$\mathrm{PMH}$ ) 的标准, 是一个集文字、图件、图片、声音、影像为一体的, 能够在网上和硬件载体 (如光盘) 上同时进行发布的 分布式数据库平台。其网上数据库系统的子系统之间以及子系统和硬件载体之间可以通过元数据获取的开放档 案倡议协议互相交换数据。

关键词 : 信息系统 数据库 数据共享

中图分类号 : Q141 文献标识码 : A 文章编号 : 1005-0094(2004)05-0553-09

\section{A model of biodiversity information integration}

QIAO Hui-Jie ${ }^{12}$, HAN Yan ${ }^{1}$, LI Nuo ${ }^{1}$, JI Li-Qiang $^{1 *}$

1 Institute of Zoology, Chinese Academy of Sciences , Beijing 100080

2 Graduate School of the Chinese Academy of Sciences ,Beijing 100039

\begin{abstract}
We proposes a model of biodiversity information integration involving data describing biodiversity entities from different layers and angles using artificial intelligence. Based on analysis of current biodiversity database systems and the demands of biodiversity researchers, this model supports decisionmaking on biodiversity conservation and sustainable use. According to the Dublin Core metadata template and the standard of the Open Archives Initiative Protocol for Metadata Harvesting (OAI-PMH) , the model builds up a platform, in both website and CDROM forms, to publish data in different data formats ( text , image , vector data , audio , and video) from distributed databases. Data exchange could be implemented smoothly among remote databases and/or between remote and local databases according to the standard of OAI-PMH.
\end{abstract}

Key words : information system , database , data sharing

\section{1 问题的提出}

据估计，世界上的生物物种约有 $500-3000$ 万 种 (Wilson et al. ,1988)。目前已定名或描述的物种 数目为 140 万种(世界资源研究所等, 1992 ; Wilson et al. ,1988) 至 170 万种 (Wilson ,1985;Tangley， 1986 ;Shen ,1987)。在中国，有高等植物 3 万多种， 脊椎动物 6347 种, 分别约占世界总数的 $10 \%$ 和 14\% ;陆生生态系统类型有 599 类 ${ }^{(1)}$ 。中国政府为
保护生物多样性积极地开展了包括建立国家协调机 制、加强立法和执法、强化就地保护、重视宣传教育、 推动全球合作等一系列卓有成效的工作, 有力地促 进了国民经济和社会的可持续发展，也为保护中国 特有的生态系统、物种资源和遗传资源做出了重要 贡献。

中国已制定和颁布了生物多样性保护法律、法 规 20 多项。到 2002 年底, 中国已建立各种类型、不 同级别的自然保护区 1757 个(不包括香港、澳门特 
别行政区和台湾省），其中国家级自然保护区有 197 个。保护区的总面积达 132.9 万平方公里, 陆地保 护区面积约占陆地国土面积的 $13.2 \%$ 。

我国研究人员已经对我国动植物和微生物资源 进行了多年的调查和研究, 先后出版了《中国植物 志》、《中国动物志》、《中国孢子植物志》等系列专 著,并增建和扩建了有关的基础研究设施, 如标本 馆、植物园、定位研究站等, 为生物多样性保护和持 续利用的研究和实践提供了大量的资料和服务 (纪 力强 , 2000)。

在生物多样性保护方面，今后任务仍相当艰巨。 为了使法规制定得更加切实可行，保护区建立得更 加科学合理,需要为决策者和科研人员提供更加科 学准确的信息。但是生物多样性的信息从数目上来 讲是海量的, 而且数据结构复杂 数据与数据之间在 不同的层次上存在着各种联系。因此，如何在这海 量的数据中有效寻找到所需的信息资源,为科研工 作和科学决策提供有价值的数据参考, 是亟待解决 的问题。

\section{2 现有的生物多样性数据集成系统}

\section{1 国外生物多样性数据集成系统}

目前，国外生物多样性数据主要集中在大的博 物馆或博物馆联盟 (如 The Natural History Meseum, NHM 和 American Natural History Museum , ANHM 等) 以及一些政府或非政府的组织机构 (如 Species 2000、Global Biodiversity Information Facility 和 Integrated Taxonomic Information System 等) (赵海军和 纪力强 2003)。

\subsection{1 物种 2000 年计划 (Species 2000 )}

物种 2000 年计划 (Species 2000 ) 是一个数据库 组织的联盟, 它为一般用户、生物学家及相关的研究 机构提供生物多样性信息服务。推动这一计划的主 要目的是建立起一个涵盖主要生物类群的数据库集 合。在各数据库中, 涉及同一生物类群的数据使用 相同的分类系统, 并尽可能多地包括该类群的所有 已知种。

数据库的组织形式采取分布式管理。现有的服 务器分布在世界各地, 而且计划在以后继续采取这 一方式。现有数据库中的数据大约涵盖了全世界 $40 \%$ 的已知物种。

到 2003 年 10 月 物种 2000 年计划已经包括了
综合分类信息系统( Integrated Taxonomic Information System ,ITIS ) 、国际豆科数据库及信息服务 ( International Legume Database \& Information Service ,ILDIS ) 等 12 个项目组( Brugman 2004)。

目前它提供以下几种服务：

（1）生物目录 (Catalogue of Life) :包含 18 个数 据库 均提供相应的检索接口，如 FishBase 等。可同 时对 260000 个物种, 420000 个同物异名 (Synonyms）,180000 个俗名，90000 篇参考文献信息进 行检索( Brugman ,2004)。各个数据库提供的信息 完备性不同。如一条在 FishBase 中的记录可以提供 科名、属名、种名以及相应标本的详细信息和照片 等,还会提供主要的参考文献、分布图等;而在 AAFC Online 中只能检索到该物种的从 界”到 种” 的分类信息 (Ruggiero，2002)；对于图片信息，可连 接到通用搜索引擎 Google ( http $/ /$ www. google. com) 上面进行简单的检索,检索到的内容并不严 谨。

生物目录还提供一个附加功能, 叫作与生物目 录的直接关联检索 (Link Directly to the Catalogue of Life)。这是 Species 2000 的主要特色，它可以通过 一个接口直接对某一物种信息在 18 个数据库中进 行检索, 接口形式为 :http $/ /$ www. sp2000. org/find. html ? genus $=($ genus name $)$ \&species $=($ species name) 返回的就是要查找的物种的相应信息。

(2) 动态名录 (Dynamic Checklist): 可根据生 物的类群，分别对微生物、真菌、植物、无脊椎动物 (暂未提供) 和脊椎动物等同一生物类群中的一个 或多个数据库进行学名的同步检索。各个类群的检 索分别有自己的搜索引擎, 并不为 Species 2000 提 供专门的接口（White 2000)。

(3) 名称服务 (Names Service): 与 Dynamic Checklist 类似，可根据生物类群，分别对微生物、真 菌、植物和脊椎动物 (暂未提供) 进行生物名称的检 索。除正式的学名外，这些名称还包括生物体非正 式的名称 或可能已经弃用的名称。各个类群的检 索分别有自己的搜索引擎, 并不为 Species 2000 提 供专门的接口。与 Dynamic Checklist 比较, 除了搜 索引擎有部分不相同外，其结构与组织形式类似 (White 2000)。

\subsection{2 世界生物多样性数据库 ( ETI-World Biodi-} versity Database ) 
分类鉴定专家中心 (Expert Center for Taxonomic Identification) 的世界生物多样性数据库 (World Biodiversity Database) ( 以下简称 ETI-WBD) 是一个 仍在持续扩大的分类学数据库。它的目标是搜集世 界上所有现存已知物种 (约 1700000 种) 的档案。 它的数据是全世界可自由访问的, 并且 ETI-WBD 允 许全世界的相关研究人员通过在线或者光盘的方式 提交自己的数据，以便供给其他人共享。

目前，ETI-WBD 有 39 个分管不同类别、不同方 向的项目组，共包括了 206812 个分类单元。有的 项目组按照地域划分，如新热带地区种属项目组 ( Neotropical Genera and Species)、荷兰植物模式标 本目录项目组 (Catalogue of the Type Specimens of the Dutch Herbaria) 等; 还有的按照功能划分, 如 Braconidae 项目组负责所有亚科的模式图。

ETI-WBD 试图提供物种层次上的尽可能多的 分类信息 (分类阶元的继承关系)、物种名、异名、描 述、模式图和参考文献等。每个分类单元还可以通 过超级链接直接在 CMBI 数据库中查寻蛋白质序 列、核酸序列、3D 结构等遗传信息。未来 WBD 还将 提供在线识别和可交互式的地理信息系统的功能。

ETI-WBD 将物种 2000 计划中的物种索引 ( Index of Species) 作为自己的虚拟的分类学参考系统， 将本身数据库中的数据与参考系统中的数据进行校 验, 以保证数据库中数据的质量。

2.1.3 全球生物多样性信息组织 (Global Biodiversity Information Facility, GBIF)

1996 年, 经济合作和发展组织( OECD) 生物信 息学大科学论坛工作组 (Mega Science Forum Working Group on Biological Informatics) 认为,尽管以往 研究工作已经取得了大量生物多样性和生态系统的 信息，但是由于信息分散且缺乏沟通渠道，这些信息 并没有发挥出应有的作用。2001 年, 全球生物多样 性信息组织 (GBIF) 在哥本哈根大学成立，作为一个 国际机构，通过互联网向广大公众免费提供生物多 样性信息( GBIF 2003)。

全球生物多样性信息组织的主要目的是将世界 各地的生物多样性数据库链接起来, 形成一个易于 利用的网络。为了使人们能够认识到研究和保护自 然资源的意义以及对自然资源巨大的经济、医学、社 会和科学潜在价值进行可持续的利用, 推广和宣传 生物多样性的信息是很有必要的(Dooley，2002)。
通过该组织主页上的 机构介绍” (Facility) 链 接，网站访问者可以了解相关的背景、宗旨和组织机 构。到目前为止，已有 21 个国家成为该机构的表决 成员, 另有 3 个国家申请成为表决会员, 10 个准会 员已同意共享生物多样性数据并正在开发提供数据 的联网服务。会员中还包括许多非政府组织，如联 合国环境规划署 (UNEP)、国际生物网 (BioNET-International)、分类鉴定专家中心等。

在 工作规划” (Work Programmes) 链接下, 全 球生物多样性信息组织以专门的页面介绍了它的 4 个主要工作领域 数据存取和数据库的互操作性、已 知生物类群名称的电子编目、生物标本信息数字化 以及能力建设。具体工作内容包括建立并维护已知 生物类群名称的电子目录, 作为分类学工作标准化 的基准数据资源, 编纂一本电子手册来指导制作标 本数字图像以便全球资源共享等。

该网站 相关链接” (Related Links)下还提供了 100 个相关网站的链接, 组织在 7 个目录下,包括： 国际公约和协定, 生物多样性搜索引擎、工具和软 件, 生物多样性研究机构, 全球专家数据库, 全球主 题数据库 相关标准 相关技术。“合作关系” ( Relationships) 链接介绍了全球多样性信息组织与 15 个 国际性和地区性机构的合作。与这些机构开展合 作, 可避免工作上的重复, 并可获得相关学科专家最 新的工作计划。以上机构包括物种 2000 年计划 (Species 2000 ) 、国际分类学数据库工作小组 (International Working Group on Taxonomic Database)、全 球迁移物种登记处 (Global Registry of Migratory Species)、欧洲环境署 (European Environmental Agency) 和泛美生物多样性信息网络 (Inter-American Biodiversity Information Network)。

\section{2 国内生物多样性数据集成系统}

\subsection{1 中国生物多样性信息系统 (CBIS)}

我国生物多样性研究具有良好的基础，据初步 估计，中国现在已收藏的生物标本约有 2600 万号， 其中中国科学院动物研究所保存了约 400 万号动物 标本, 中国科学院植物研究所保存了约 300 万号植 物标本, 另外还有中国植被图 (1/400 万, $1 / 100$ 万) 等一批十分重要的图件。遍布全国的生物标本馆、 水族馆和种质资源库等保存了大量的相关信息。中 国生态系统研究网络 (CERN)、自然保护区和植物 园等研究和保护基地也是重要的生物多样性数据源 


\section{(纪力强 , 2000)。}

在现有资源的基础上, 中国科学院建立了中国 生物多样性信息系统 (CBIS)，这一系统能够为国家 和地方各级决策部门提供初步的生物多样性科学数 据, 同时可以在全社会范围内普及生物多样性知识。

中国生物多样性信息系统在内容上由基础数据 库、模型库和专家系统库组成。基础数据库中的数 据包括 物种编目、濒危和保护物种、典型生态系统、 标本、移地保护、就地保护、种子和种质资源、环境因 子及植被、有关的社会和经济发展的数据、文献信息 和生物多样性信息编目的数据等。中国生物多样性 信息系统的模型包括生态系统中关键种的种群动态 模型、时空动态的种群生存力分析模型、生物多样性 状态评价模型、建模环境和工具。中国生物多样性 信息系统的专家系统包括濒危物种保护专家系统、 自然保护区规划专家系统、生物多样性资源持续利 用专家系统、专家系统开发环境和工具等几方面内 容。

目前，CBIS 已经建立了上百个生物多样性方面 的数据库，包括物种编目、濒危物种、标本数据库、生 态系统和保护区经济动物植物和微生物等 6 大类 36 个小类的数据库, 总记录数已经超过百万条, 大 部分数据库已经上网, 可以通过网络在线查询数据 ( http $\because /$ cbis. brim. ac. cn)。

\subsection{2 中国物种信息系统 (CSIS)}

由中国环境与发展国际合作委员会生态安全课 题组 (原国合会生物多样性工作组) 建立，其目标是 建立一个综合的中国生物多样性数据库，作为生物 多样性计划、发展、生物编目和保护的基本数据库。 目前中国物种信息系统 (CSIS ,中、英文版) 已经建 立, 并能在互联网上查阅 (www. chinabiodiversity. com)。

该系统已经有超过 8900 个种和亚种的信息，包 括分类、同物异名、濒危等级、中文名称、英文名称、 商用名称、分布和数量、产地、所面临威胁、已采取的 保护措施、意见建议情况。现在正在 3 个不同的层 次上发展 : (1) 收集基础信息, 为生物多样性编目调 查提供工具，包括提供物种信息和鉴定工具，并通过 专家和保护区网络促进编目工作的开展 ; (2) 促进 国际国内信息共享，包括与国际国内的相关组织合
作 ,并将信息发布到互联网; ; 3 )根据信息系统中的 信息, 发展生物多样性分析和评估手段, 为国家和地 区的生物多样性保护规划提供信息和建议。

该系统还收集和整理了中国 1000 多个自然保 护区的相关信息，包括 联系地址、介绍、地图、物种 名录、代表性生态系统照片、野生动植物的野生生活 照片以及生态旅游相关信息，建立了中国自然保护 区网络。还收集和整理了中国生物多样性研究和管 理专家的信息，包括联系地址和专长 建立了中国生 物多样性专家信息网络。

已经完成的研究课题有:中国与周边国家或地 区野生动物贸易的种类和数量 (http $\% /$ www. chinabiodiversity. com/jij. html)、中药和野生动物利用水 平和生物多样性产品与服务给中国经济所带来利益 的研究。目前正在进行的课题有:高山草地保护研 究、入侵物种、森林恢复中涉及的生物多样性保护问 题研究、鸟类和哺乳类野外工作手册等。该网站收 录的大多数为我国濒危物种的信息。

\section{3 国内外集成系统的比较}

表 1 对上面介绍的几个数据库系统和其他利用 率比较高的生物多样性数据库的数据特点作了一些 比较。

通过比较可以看出，不同的系统采取的集成策 略不同。总的来说有三种策略 :(1) 信息系统自身 带有数据库, 数据检索主要从自身的数据库中读取, 如 ETI-WDB、ILDIS、CBIS、CSIS 等;(2) 信息系统自 身没有任何数据, 所有数据均从其他系统的数据库 中读取。如 Species 2000 等; (3) 自身有数据库, 同 时从别的信息系统中读取自身数据库中没有的数 据。如 FishBase、CephBase 等。

表 2 比较了部分信息系统提供的相应工具和一 些其他特征，如提供离线检索的工具、地理信息系统 的图件等。

目前 提供导出/下载数据工具的信息系统很 少,同时下载的信息有各种限制，而且现有的信息仅 限于导出或者下载, 还没有一个信息系统能做到数 据直接上传或者在客户端实现数据交换。另外,基 于网络的地理信息系统 (Web GIS) 的应用也很少， 使用很简单。国内的生物多样性信息系统尚未提供 除了信息检索之外的任何其他工具。 
表 1 每个生物多样性数据库包含的数据项

Table 1 Major contents in current biodiversity information systems

\begin{tabular}{|c|c|c|c|c|c|c|c|}
\hline \multirow[b]{2}{*}{$\begin{array}{c}\text { 生物多样性数据库 } \\
\text { Biodiversity Databases }\end{array}$} & \multicolumn{7}{|c|}{ 生物多样性数据 Biodiversity Data } \\
\hline & $\begin{array}{l}\text { 标本信息 } \\
\text { Collection } \\
\text { information }\end{array}$ & $\begin{array}{l}\text { 图片 } \\
\text { Image }\end{array}$ & $\begin{array}{l}\text { 图件 } \\
\text { Map }\end{array}$ & $\begin{array}{c}\text { 生物学描述 } \\
\text { Biological } \\
\text { description }\end{array}$ & $\begin{array}{c}\text { 保护方式 } \\
\text { Conservation } \\
\text { method }\end{array}$ & $\begin{array}{c}\text { 生境/生态系统 } \\
\text { Habitat/eco- } \\
\text { system }\end{array}$ & $\begin{array}{l}\text { 基因信息 } \\
\text { Genetic } \\
\text { information }\end{array}$ \\
\hline Species 2000 & {$[\mathrm{X}]$} & {$[\mathrm{X}]$} & {$[\mathrm{X}]$} & {$[\mathrm{X}]$} & {$[\mathrm{X}]$} & {$[\mathrm{X}]$} & {$[\mathrm{X}]$} \\
\hline ETI-WDB & & $\mathrm{X}$ & $\mathrm{x}$ & $\mathrm{X}$ & $\mathrm{X}$ & $\mathrm{X}$ & \\
\hline ITIS & $\mathrm{X}$ & & & $\mathrm{X}$ & $\mathrm{X}$ & $\mathrm{X}$ & $\mathrm{X}$ \\
\hline FishBase & & $\mathrm{X}$ & $\mathrm{X}$ & $\mathrm{X}$ & $\mathrm{X}$ & $\mathrm{X}$ & {$[\mathrm{X}]$} \\
\hline CephBase & $\mathrm{X}$ & $\mathrm{X}$ & $\mathrm{X}$ & $\mathrm{X}$ & & & {$[\mathrm{X}]$} \\
\hline ILDIS & & & $\mathrm{X}$ & $\mathrm{x}$ & $\mathrm{X}$ & & \\
\hline CBIS & $\mathrm{X}$ & $\mathrm{X}$ & $\mathrm{X}$ & $\mathrm{X}$ & $\mathrm{X}$ & $\mathrm{X}$ & \\
\hline CSIS & & $\mathrm{X}$ & $\mathrm{X}$ & $\mathrm{X}$ & $\mathrm{x}$ & & \\
\hline
\end{tabular}

$\mathrm{X}$ 表示该数据库包含的内容, $[\mathrm{X}]$ 表示该数据库提供了向其他数据库数据源的超级链接

$\mathrm{X}$ represents data provided , [X] represents a hyper-text link to other data source provided.

表 2 不同生物多样性数据库提供的辅助工具和其他特征对比表

Table 2 Supplementary tools and features provided by current biodiversity information systems

\begin{tabular}{|c|c|c|c|}
\hline \multirow{2}{*}{$\begin{array}{l}\text { 生物多样性数据库 } \\
\text { Biodiversity databases }\end{array}$} & \multicolumn{3}{|c|}{ 数据库工具/特征 Database tools/features } \\
\hline & $\begin{array}{l}\text { 导出数据 } \\
\text { Export data }\end{array}$ & $\begin{array}{c}\text { 实时产生地理图件 } \\
\text { Map generation based on real-time data }\end{array}$ & $\begin{array}{l}\text { 其他特征 } \\
\text { Other features }\end{array}$ \\
\hline Species 2000 & & & $\begin{array}{l}\text { 提供生物目录的直接关联检索 } \\
\text { 工具 The name service tools }\end{array}$ \\
\hline ETI-WDB & $\mathrm{x}$ & & \\
\hline ITIS & & & \\
\hline FishBase & $\mathrm{X}$ & $\mathrm{X}$ & \\
\hline CephBase & $\begin{array}{c}\mathrm{X} \\
\text { Species name list }\end{array}$ & & \\
\hline ILDIS & $\begin{array}{c}\mathrm{X} \\
\text { Species name list }\end{array}$ & & \\
\hline CBIS & & & \\
\hline CSIS & & & \\
\hline
\end{tabular}

$\mathrm{X}$ 表示该系统包含的工具。X represents tool/feature provided

\section{3 生物多样性数据的多源性}

3.1 生物多样性数据是一个多源性的空间数据 类似于其他具有空间属性的数据，生物多样性 数据的多源性主要表现在以下几个方面 ( Devogele et al. , 1998)。

3.1 .1 多层次、多角度性

生物多样性信息指的是生态系统中的各种信 息，生态系统中研究对象的多种类特点决定了生物 多样性信息的多层次、多角度性。对于同一个区域， 虽然在实际空间中其几何特征是一致的，但却可以 从多个层次和角度对它进行描述，如从生物学角度 就会有物种信息、群落信息等多个层次 ; 从地理学角
度有地理位置、海拔高度、气候、地貌、土壤等自然地 理特征，还包括经济社会信息，如行政区区域、人口、 经济活动等 (Bennett , 1997)。

\subsection{2 多时空性和多尺度}

生物多样性数据具有很强的时空特性。一个生 态系统中的数据源既有同一时间不同空间的物种分 布数据，也有同一空间不同时间序列的数据，如生态 系统演替的数据;既有以米甚至厘米计量的小生境 数据, 也有以公里甚至更大尺度计量的景观数据。

\subsection{3 获取手段的多源性}

根据分析目的的需求和技术条件的限制，获取 生物多样性数据的方法有多种多样, 包括来自现有 数据系统、图表、遥感手段、GPS 手段、统计调查、实 
地勘测等。这些不同手段获得的数据的存储格式、 提取和处理手段都各不相同(Bishr，1998)。

\subsection{4 存储格式的多样性}

根据各地的条件不同和表述方法不同，生物多 样性信息可能存在多种不同的存咜格式。如何将多 种格式集成起来, 使之能够向使用者提供综合的信 息,也是要讨论的问题。

3.2 多源性生物多样性数据处理方式

国际上对于多源性数据集成的方式主要有下面 三种 数据格式转换模式、数据互操作模式和直接数 据访问模式 (Arctur et al. , 1998)。

(1) 数据格式转换模式:在这种模式下，各种数 据格式经专门的数据转换程序进行格式转换后, 复 制到集成系统中的数据库或文件中。这种模式的优 势在于数据的格式规范、访问简单、集成度高。并 且，在 Microsoft OS 的平台下面，提供了 ODBC(开放 数据接口, Open Database Connectivity)、DCOM (分 布式的组件对象模型，Distributed Component Object Model) ,允许在统一的数据访问与应用程序接口上 有一定的拓展, 即在一定程度上允许数据与应用有 差异。该模式的缺点在于数据更新不方便, 而且每 次复制过程都会花费一定的时间，更新也无法做到 实时性。

(2) 数据互操作模式 :互操作是指在异构数据 库和分布式计算的情况下，在相互理解的基础上，系 统的各个部分之间能透明地获取所需信息。这种应 用需要在提供数据源( 数据服务器, Data Servers) 的 一端设置访问权限和访问规则。客户端( 数据客 户, Data Clients) 使用某种数据的过程就是发出数 据请求, 由数据服务器提供服务, 其最终目的是使数 据客户能根据其拥有的权限访问一定范围的由数据 服务器提供的数据。与数据格式转换模式相比，这 种形式能够支持更大范围的数据格式异构, 但是需 要对系统进行复杂的设计和严格的权限设定，才能 保证服务的安全性( 钟扬等,2000)。

(3) 直接数据访问模式: 指在系统的一个部分 中实现对其他数据格式的直接访问。直接数据访问 避免了繁琐的数据转换，提供了一种更为经济实用 的多源数据集成模式。但是数据格式本身的异构会 导致信息的不完整，而且要对每一个数据格式编写 相应的代码进行访问，会直接限制系统的规模。

\section{3 都柏林核心 ( Dublin Core)}

都柏林核心可以作为数据格式转换模式的格式 基础。都柏林核心是国际组织 Dublin Core Metadata Initiative 拟定的用于标识电子资源的一种简要目录 模式。都柏林核心参考了图书馆卡片目录的模式, 制定了 15 项广义的元数据, 通过元数据可以反映电 子资源的目录信息。以都柏林核心为基础, 将多种 格式的多源数据都转化成统一的格式, 就可以方便 地进行数据交换。

\subsection{OAI 协议 (Open Archives Initiative Protocol)}

OAI 协议是 1999 年由美国数字图书馆联盟 (DLF)、网络信息联盟 $(\mathrm{CNF})$ 等组织提出的一个应 用框架。最初是为了解决学术性电子化预印本 (electronic pre-print) 及相关文献资料 (Archives) 的互 操作问题而提出的。2000 年, OAI 协议的应用扩展 到数字图书馆领域，目的是实现分散的、不同系统平 台之间的元数据的交换和共享, 提高系统的互操作 能力。

\section{4 数据集成模式初探}

进行数据集成的目的,主要是为了使分类学家 和生态学家能够方便地使用生物多样性数据。所 以, 在设计系统初期要从他们需求的角度来考虑。 经过调研后发现，国内的专家更多地使用国外的相 关数据库, 而且利用率在逐渐提高。但国外的数据 库并不着重于国内物种信息的集成，因而存在着数 据不全、缺少足够的辅助信息 (如分布地信息) 等缺 陷。因此，集成模式既要借鉴国外现有的成功做法， 还要充分考虑中国的现状和需求特点。

\section{1 集成的模式探讨}

综合考虑各种因素,我们提出了一个包含多层 次多角度数据、并带有一定人工智能的生物多样性 集成数据库平台的构想。所谓的多层次，就是对每 一个能够检索的对象在遗传、物种、生态系统、景观 等多个层次上进行描述，尽量做到信息的最大集成。 多角度就是指从数据源、定名人和栖息地等多个角 度 对同一个检索对象加以描述。在这些描述的基 础上，再加入一些与该检索对象相关的联想信息，帮 助不熟悉信息系统的用户获得更加完备的信息，具 有一定的人工智能特性。

在建立数据描述的基础上，加入现有的 Web GIS (基于网络的地理信息系统) 技术,对信息系统 进行二次开发，建立一个集文字、图件、图片、声音、 
影像为一体的, 能够在网上和硬件载体 (如光盘) 上 同时进行发布的数据库平台。

\section{2 数据源的选择}

并非所有的数据都适合集成到拟建立的信息系 统中，以下几种情况就不适合进行数据集成：

(1) 数据量非常少, 与其他数据联系不紧密, 没 有必要集成到系统中。

（2）现有的技术手段很难或尚无法完成集成工 作。比如一些特殊的原始文献信息，本身的格式比 较复杂, 有经验的人可以从中获得所需的信息, 但是 通过计算机就很难进行有效的分析和检索。

(3) 数据集不完整, 对科学研究没有实际意义。

因此，集成进入信息系统的数据源必须具有以 下几个特征 :

(1) 数据量足够大。单凭人力检索难以查找到 所需的资源，或者虽可以查找到但要花费大量的时 间。而通过集成系统就可以提高显著数据使用者的 工作效率。

(2) 与其他数据关联紧密。即使数据量很小, 但被检索的频度很高, 可以显著提高检索系统的综 合工作效率。

(3) 数据完整 格式比较规范。

根据上面的描述，以下的一些数据可作为优先 考虑的对象：

(1) 在物种层次上 现有生物多样性信息系统 中的数据，包括物种信息、标本信息、保护区信息以 及相关文献信息等。

(2) 在生态系统层次上, 可将数据信息比较系 统和完整的重点地区 (如东灵山地区和神农架地区 等) 作为首先加入信息系统的数据, 进行重点建设 规划。

\section{3 数据处理}

现在国内生物多样性数据的情况是 现已数据 化的资源大部分集中在几个数据源点, 并且大都是 在 Windows 平台下实现的 格式比较统一，进行格式 转换和相互访问比较方便, 没有上网的还都以文本 的方式记录。因此, 最适合数据集成的形式应该是 数据格式转换模式和数据互操作模式的结合。

在这种结合的模式下, 可将现存多种数据格式 经专门的数据转换程序, 按照都柏林核心的标准, 在 各个数据源点进行格式转换, 并分别存放在源点的 数据库中, 每个源点的数据库格式是相同的。发布
系统在一个单独的节点，它不隶属于任何一个数据 源点, 但含有所有数据源点的配置信息, 可以通过统 一的接口最大限度地访问各个数据源点的所有数据 资源。这样，发布系统对每一个数据源点的访问方 式是相同的，访问简单，也可以很方便地从其他数据 源点通过统一的接口获得所需要的信息，集成度高； 每一个源点各自管理自己的数据，更新之后的数据 在发布系统处可以同时得到体现，继承了数据互操 作模式的数据实时更新的优点。通过这种分布式组 织结构, 可以最大限度地做到统一格式的高集成性 和分布存储的高度灵活性，集中控制、分散管理。

同时，各个数据源点之间还可以实现数据交换， 采用基于开放文献预研的元数据互操作协议 (Open Archives Initiative Protocol for Metadata Harvesting, $\mathrm{OAI}-\mathrm{PMH})$ 在统一设计的相同平台、相同介质的分 布式数据库之间进行。不仅如此, $\mathrm{OAI}$ 协议还可以 实现跨平台的数据交换。所以, $\mathrm{OAI}$ 协议的应用也 为集成系统的进一步扩展打下了基础。

\section{4 集成的方式}

根据需求分析、国内用户的使用习惯和国际上 生物多样性信息系统发展的趋势，拟建立的集成系 统将采用两种集成形式面向最终的使用者：

\subsection{1 网站形式}

这种形式是最常见的集成与发布形式。集成模 式采取汶览器/服务器 (Brower/Server) 模式。B/S 模式是一种以 Web 技术为基础的 MIS 系统平台模 式，它把传统 $\mathrm{C} / \mathrm{S}$ 模式中的服务器部分分解为一个 数据服务器与一个或多个应用服务器 (Web 服务 器)，从而构成一个三层结构的客户服务器体系。 它简化了客户端,也简化了系统的开发和维护。各 个用户通过 Http 请求在权限范围内调用 Web 服务 器上的不同处理程序, 从而完成对数据的查询或修 改。

集成系统的 $\mathrm{B} / \mathrm{S}$ 模式需要的后台数据服务器 采取分布式关系方式。服务器分布于各个数据源 点，存储格式统一,由统一的入口管理；数据发布系 统就是一个 Web 服务器, 遵循 OAI-PMH 协议从各 个数据源点中获取数据, 经过整合, 并按照不同的权 限 (即不同权限的使用者能看到的内容不同) 将数 据的全部或者部分发布给使用者。

这种形式的主要使用对象是生物学方面的专家 和生物学爱好者。使用者不需要安装特殊的软件, 
只要上网就可以索取他们所需要的资料。但是, 这 种方式不能进行进一步的订制开发，不能进行特殊 的分析、计算等，所以，对于科学研究的专家来讲，功 能尚嫌单薄。

为了解决这个问题,可以采取下面的集成形式 作为补充。

\subsection{2 硬件载体形式}

这种发布形式以光盘为载体,内容主要包括两 部分 数据和软件包。数据指的就是软件系统所需 的基本数据和为特定目的而收集的学科数据，它可 以是数据源点数据的一部分或者全部数据, 或者是 专家自己的数据，按照规定的格式存储。软件包指 的是一系列应用程序的集合，如生物多样性分析和 评价的工具软件等, 它们可以利用本地( 光盘载体 和个人计算机内) 的数据, 实现数据读写、分析、统 计等功能, 也可以与一个或多个数据源点的数据库 交互, 将本地和远程的数据结合起来分析处理, 实现 分布式存储的数据的综合利用。

这种方式可以允许生物多样性研究者在客户端 根据自己的需要、遵循统一的数据存储和交换格式 进行二次开发, 还可以以软件包的方式共享网上公 开的数据，以弥补网上发布的数据分析功能少的缺 陷。而且, 研究者可以根据自己的需要下载和保存 网上开放的数据，也可以通过协商与其他研究者共 享尚未开放的数据, 而不必担心自己的数据在互联 网上公开。

通过第一种形式, 可以很好地解决公共性的生 物多样性基础数据的发布问题，而第二种形式可以 尽可能大地扩充数据源的范围，给使用者提供更大 的综合空间，实现更多的分析功能。二者的数据基 础都是基于都柏林核心的存储格式, 交互方式都遵 循 OAI-PMH 协议。在统一的协议下面，二者可以很 好地协同工作。

\section{5 集成应用的展望}

通过本文研究和设计的模式，可以对现有的分 布式或集中式的生物多样性信息/数据库系统进行 改造，以方便用户的数据检索。对于分布式的系统 (如 CBIS 系统等)，可以通过格式转换的方式统一 同类数据库的结构 将相关的数据归并或建立索引, 并构造功能一致的分布式查询门户界面，从而可以 增加系统集成度，提高用户的查询效率。对于相对 独立的数据库系统, 如 CBIS 内部的一些单一数据源
的数据库 或者是 CBIS 的物种数据库与 CSIS 系统 之间, 可以尝试采用 OAI-PMH 的方式进行数据交 换,为用户提供一站式的数据服务。

\section{5 总结}

本文根据现有生物多样性数据库系统的数据特 点, 参考国内外成功的集成系统经验,并借鉴了计算 机科学技术、图书馆学等相关学科和领域的技术规 范, 提出了网站发布与客户端软件包相结合的数据 集成方式。其中,网站发布方式的后台数据库采取 分布式存储，遵循都柏林核心的数据存储标准，发布 系统与数据源点的数据交换遵循 OAI-PMH 协议; 客 户端软件包为一个遵循 OAI-PMH 协议的开放系统， 提供数据读写，数据交换等基本功能，生物多样性工 作者可以在 OAI-PMH 协议的基础上 根据自己的需 要对客户端软件进行二次开发, 满足科研工作的需 求。网站和客户端软件包可以交换数据,客户端软 件包之间也可以直接交换数据。通过这种方式, 达 到数据共享的目的，从而逐渐发展成为一个集文字、 图件、图片、声音、影像为一体,能够在网上和硬件载 体 (如光盘) 上同时进行生物多样性信息发布的分 布式数据库平台。

\section{参考文献}

Arctur, D., Hair, D. and Timson, G. 1998. Issues and prospects for the next generation of the spatial data transfer standard (SDTS). Geographical Information Science, 12: $403-425$.

Bennett, D. A. 1997. A framework for the integration of geographical information systems and modelbase management. Geographical Information Science, 11: 337 - 357.

Bishr, Y. 1998. Overcoming the semantic and other barriers to GIS interoperability. International Journal of Geographical Information Science, 12: 299 - 314.

Brugman, M. L. 2004. The Species 2000 Project. Updated at http: //www. species2000. org/sp2000org. html.

Devogele, T., Parent, C. and Spaccapietra, S. On spatial database integration. International Journal of Geographical Information Science, 12: 335 - 352 .

Dooley, E. E. 2002. Global biodiversity information facility. Environmental Health Perspectives, 110: A666 - A669.

GBIF. 2003. GBIF Work Programme 2004. Approved by the GBIF Governing Board at GB7 October 2003. Tsukuba, Japan. 
Ji, L. Q. (纪力强). 2000. The construction status of biodiversity information system and a brief introduction of CBIS. Chinese Biodiversity (生物多样性), 8: 41 - 49. ( in Chinese with English abstract)

Ruggiero, M. 2002. The Interagency Taxonomic Information System. Updated at http: //www. itis. usda. gov/info. html.

Shen S. 1987. Biological diversity and public policy. BioScience. 37: $709-712$.

Tangley, L. 1986. Biological diversity goes public. BioScience, 36: $708-711$.

White, R. 2000. Search the Dynamic Checklist. Updated at http: //www. species2000. org/dynamicchecklist. html

Wilson, E. O. 1985. The biological diversity crisis. BioScience, 35: $700-706$.

Wilson, E. O., Frank, B. and Baird, J. 1988. The current state of biological diversity. In: Wilson, E. O. ( ed. ). Biodiversity. National Academy of Sciences Press, Washington, D. C. , 3-18.

World Resources Institute (世界资源研究所). 1992. Global Biodiversity Strategy (全球生物多样性策略). Standards Press of China, Beijing.

Zhao, H. J. (赵海军) and Ji, L. Q. (纪力强). 2003. Biodiversity assessment at broad scale. Biodiversity Science (生物多 样性), 11:78 - 85. (in Chinese with English abstract)

Zhong, Y. (钟扬), Zhang, L. (张亮), Ren, W. W. (任文伟) and Chen, J. K. (陈家宽) . 2000. Biodiversity information: a new direction of bioinformatics and biodiversity science and related key techniques. Chinese Biodiversity(生物 多样性), 8: 397 - 404. ( in Chinese with English abstract)

(责任编辑 : 时意专)

\section{《生物多样性》2005 年征订启事}

《生物多样性》1993 年创刊,由中国科学院生物多样性委员会、中国科学院植物研究所、动物研究所、微生物研究所共同主 办, 是全面报道动物、植物、微生物在生物多样性各研究领域的综合性学术刊物。主要报道范围: (1) 生物多样性理论和应用研 究的原创性学术论文; (2) 具有新观点和前瞻性的综述; (3) 国家和地方的重要或重大生态工程对生物多样性保护及可持续利 用的影响及其风险评估; (4) 外来种入侵及遗传改性生物体的释放对本地生物多样性的影响及其监控措施。

《生物多样性》被国内外多家检索系统收录 影响因子和被引频次逐年上升 影响因子在国内生物学刊物中始终位居前 10 位 2002 年影响因子在生物类期刊中排名第五 (据中国科技信息研究所《2003 年版中国科技期刊引证报告》)。出版周期保持 在 7 个月之内 报道及时, 可读性强。自 1993 年创刊以来刊登的所有论文均已实现全文上网 ( http $/ /$ www. brim. ac. cn ,http $\because /$ www. biodiversity-science. net) ,可供读者免费汶览和下载全文。

《生物多样性》为大 16 开本, 88 页, 双月刊, 全年 6 期, 全铜版纸印刷, 国内刊号:CN11-3247/Q ,邮发代号 $82-858$,国际刊 号 :ISSN1005-0094 海外发行代号 1395BM 海外发行机构 :中国国际图书贸易总公司。每期定价 28 元, 全年 168 元 (含邮寄 费)。国内外公开发行，全国名地邮局均可订阅。

请广大读者及时订阅，并欢迎踊跃投稿！

地址 : 100093 北京香山南辛村 20 号《生物多样性》编辑部

电话 :(010)82598376,010)62591431-6137 E-mail : biodiversity@ ibcas. ac.cn 